Research Article

\title{
Knaster-Kuratowski-Mazurkiewicz Theorem in Generalized Metric Spaces with Applications
}

\author{
Nayyar Mehmood ${ }^{D},{ }^{1}$ Israr Ali Khan, ${ }^{2}$ Haris Latif, ${ }^{1}$ and Niaz Ahmad ${ }^{1}$ \\ ${ }^{1}$ Department of Mathematics and Statistics, International Islamic University, Islamabad, Pakistan \\ ${ }^{2}$ Department of Mathematics, Namal University, Mianwali, Pakistan \\ Correspondence should be addressed to Nayyar Mehmood; nayyarmaths@gmail.com
}

Received 26 August 2021; Accepted 26 October 2021; Published 11 November 2021

Academic Editor: Nawab Hussain

Copyright (C) 2021 Nayyar Mehmood et al. This is an open access article distributed under the Creative Commons Attribution License, which permits unrestricted use, distribution, and reproduction in any medium, provided the original work is properly cited.

\begin{abstract}
We study Knaster-Kuratowski-Mazurkiewicz theorem in the setting of generalized metric spaces. We establish some results on fixed points of Knaster-Kuratowski-Mazurkiewicz (KKM) mappings. Fan's matching and Schauder's type fixed point theorem in generalized metric spaces are also proved as interesting consequences of our main results. Examples are given to validate our results. We use these results to prove existence result for a given Atangana-Baleanu-Caputo fractional boundary value problem.
\end{abstract}

\section{Introduction}

In the large spectrum of mathematical problems, a fixed point of a specific map provides the solution of a mathematical problem. Therefore, fixed point theory is of pronounced significance in numerous fields of mathematics and other disciplines of science and engineering. Fixed point results describe the conditions under which a mathematical problem has a solution. Some fixed point results can be seen in [1-3].

The notion of metric space is fundamental in mathematics and has a major role in understanding and applying the topological concepts in different domains of analysis. This idea has pulled a substantial consideration from mathematicians owing to the notion of fixed point theory in metric spaces. Numerous extension and generalizations of the notion of metric space have been done in the literature. Czerwik [4] in 1993 presented the idea of b-metric spaces. In 1998, Czerwik [5] reintroduced this idea in which the constant 2 associated with the triangular inequality was replaced by a constant $k \geq 1$. In 2010, Khamsi and Hussain [6] generalized the idea of b-metric and named it metric type spaces. After that Hitzler and Seda [7] in 2000 gave the idea about dislocated metric spaces in which distance of a point from itself may or may not be zero. The idea of generalized metric spaces was given by Jleli and Samet [8], which covers distinctive notable structures including metric type spaces, metric spaces, and dislocated metric spaces, among others.

KKM map was presented by Knaster et al. [9] in 1929 and it established the framework for some notable existing results like Ky Fan Browder's fixed point theorem, Nash's equilibrium theorem, and Ky Fan's minimax inequality theorem [10-14]. The Fan's theorem [15] is a significant result for KKM mappings and is being implemented as a useful technique in the modern nonlinear analysis. The first endeavor to stretch out these types of theorems in metric spaces was done in [16], where the author studied the case of hyperconvex metric spaces. Using the work of Chang and Yen [17] and the idea of Khamsi [16], Amini et al. introduced KKM mappings in metric spaces [18]. After that, Khamsi and Hussain [6] extended those results (of [18]) in the settings of metric type spaces.

In Section 2 of this article, we give some basic definitions and notions for the sake of completeness. We also define and study open set in generalized metric spaces and show that an open ball needs not to be an open set in general. In Section 3, we extend the theorems and the related results of [6] to generalized metric spaces. We also prove some results related to the fixed points of KKM mappings in generalized metric 
spaces in this section. Fan's matching and Schauder's type fixed point theorem as interesting consequences of our main results are furnished in Section 4.

\section{Basic Definitions and Results}

In this section, we recall some basic definitions, which we use to prove our main results.

Definition 1 (see [8]). Let $W \neq \phi$ and $d: W \times W \longrightarrow[0, \infty]$ be the given mapping. For every $\lambda \in W$, define the following set:

$$
C(d, W, \lambda)=\left\{\left\{\lambda_{n}\right\} \subset W: \lim _{n \longrightarrow \infty} d\left(\lambda_{n}, \lambda\right)=0\right\}
$$

Definition 2 (see [8]). Let $W \neq \phi$ and $d: W \times W \longrightarrow[0, \infty]$ be the given mapping. Then, $d$ is known as a generalized metric on $W$, if for all $\beta, \lambda \in W$, the following conditions are satisfied:

(1) $d(\lambda, \beta)=d(\beta, \lambda)$

(2) $d(\lambda, \beta)=0 \Rightarrow \lambda=\beta$

(3) For $\left\{\lambda_{n}\right\} \in C(d, W, \lambda)$, there exists $S>0$ such that

$$
d(\lambda, \beta) \leq S \lim _{n \longrightarrow \infty} \sup d\left(\lambda_{n}, \beta\right)
$$

The pair $(W, d)$ is known as a generalized metric space.

Clearly, if the set $C(d, W, \lambda)=\phi$ for every $\lambda \in W$, then $(W, d)$ is a generalized metric space if and only if Equations (35) and (44) are satisfied.

Definition 3 (see [8]). Let $\left\{\lambda_{n}\right\}$ be the sequence in the generalized metric space $(W, d)$. Then, $\left\{\lambda_{n}\right\}$ is $d$-Cauchy sequence if

$$
\lim _{p, q \longrightarrow \infty} d\left(\lambda_{p}, \lambda_{p+q}\right)=0
$$

Definition 4 (see [8]). Consider the generalized metric space $(W, d)$ and $\lambda \in W$. The sequence $\left\{\lambda_{n}\right\}$ in $W$ is $d$ which converges to $\lambda$ if

$$
\left\{\lambda_{n}\right\} \in C(d, W, \lambda)
$$

Recall that open and closed balls in the generalized metric space $(W, d)$ are, respectively, defined as $B(\alpha, r)=\{\beta \epsilon$ $W: d(\alpha, \beta)<r\}$ and $B[\alpha, r]=\{\beta \in W: d(\alpha, \beta) \leq r\}$ for any $\alpha$ $\in W$ and $r>0$. Now, we define open set in a generalized metric space similar as defined in [6].

Definition 5. A nonempty subset $Y$ of the generalized metric space $(W, d)$ is said to be open if for any $\kappa \in Y$, there exists $\varepsilon>0$ such that

$$
B(\kappa, \varepsilon) \subseteq Y
$$

In this case, we denote $\kappa \in Y^{\circ}$ and read it as $\kappa$ which is the interior point of $Y$. The collection of all such subsets of $W$ will be denoted by $\tau$, which defines topology on $(W, d)$. The complement of an open set is called closed set and if $\kappa$ $\in \bar{Y}$ is called closure of $Y$; then, $\kappa \in Y$ or there exists $\left\{\kappa_{n}\right\}$ $\in Y$ such that $\left\{\kappa_{n}\right\} \in C(d, W, \kappa)$.

Remark 6. It is not necessary for an open ball in a generalized metric space to be an open set.

Example 1. Let $P=[0,1 / 2], Q=(1 / 2,1]$, and $W=P \cup Q$. Define $d ; W \times W \longrightarrow[0, \infty]$ by

$$
d(\alpha, \beta)=d(\beta, \alpha)= \begin{cases}0, & \alpha=\beta \\ 1, & \alpha \neq \beta, \alpha, \beta \in P, \text { or } \alpha, \beta \in Q \\ \alpha, & \alpha \in P, \beta \in Q\end{cases}
$$

Then, $(W, d)$ is the generalized metric space. Now, the open ball having center $\alpha_{\circ}=1 / 2$ and radius $\delta=1$, denoted by the set $A$, is given by the following:

$$
A=B\left(\frac{1}{2}, 1\right)=\left[\frac{1}{2}, 1\right] \text {. }
$$

Choose $1=\gamma \in A$ and $\delta^{\prime}>0$, then

$$
B\left(1, \delta^{\prime}\right)=\left\{\alpha \in W: d(1, \alpha)<\delta^{\prime}\right\}
$$

For any $\delta^{\prime}>0$, we have infinite many $\alpha \in P$ such that $\alpha$ $\in B\left(1, \delta^{\prime}\right)$. So, there does not exist $\delta^{\prime}>0$ satisfying

$$
B\left(1, \delta^{\prime}\right) \subseteq A
$$

Thus, the open ball $A=B(1 / 2,1)$ is not an open set.

Definition 7. Consider the nonempty subset $Y$ of the generalized metric space $(W, d)$. Then, $Y$ is referred as sequentially compact if there exists convergent subsequence $\left\{\alpha_{n_{k}}\right\}$ for every sequence $\left\{\alpha_{n}\right\}$ in $Y$. $Y$ is called compact if $Y$ is sequentially compact. $Y$ is totally bounded if for any $\varepsilon>0$, we have $\gamma_{i} \in Y, 1 \leq i \leq p$ such that

$$
Y \subseteq \bigcup_{i=1}^{p} B\left(\gamma_{i}, \epsilon\right)
$$

\section{Main Results}

We start with some useful notions which are essential to establish our main results.

Let $Y$ and $Z$ be two topological spaces and $G: Y \longrightarrow 2^{Z}$ be the nonempty set-valued mapping, where $2^{Z}$ represents the collection of all nonempty subsets of $Z$.

The set-valued mapping $G: Y \longrightarrow 2^{Z}$ is referred to be as follows: 
(i) Closed if the graph $\operatorname{Gr}(G)=\{(y, z) \in Y \times Z ; z \in G(y$ )\} is closed

(ii) Compact if $\overline{G(Y)}$ is compact in $Z$

The set of all nonempty finite subsets of a set $W$ is denoted by $\langle W\rangle$. For a nonempty bounded subset $Y$ of the generalized metric space $(W, d)$, we define the following:

$c o(Y)=\cap\{D \subset W$, where $D$ is closed ball in $W$ containing $Y\}$

and $Y$ is admissible in $W$, if

$$
\operatorname{co}(Y)=Y
$$

i.e., $Y$ is admissible if the intersection of all closed balls $D$ containing $Y$ is $Y$. If for any $D \in\langle Y\rangle$,

$$
\operatorname{co}(D) \subset Y
$$

then $Y$ is a subadmissible subset of $W$. Clearly, if $Y$ is admissible in $W$, then $Y$ is also subadmissible.

Consider the subadmissible subset $Y$ of the generalized metric space $(W, d)$. A set-valued mapping $G: Y \longrightarrow 2^{W}$ is known as KKM mapping if

$$
\operatorname{co}(A) \subset G(A)
$$

for any $A \in\langle Y\rangle$. More generally, for the topological space $Z$, consider the two set-valued mappings $G: Y \longrightarrow 2^{Z}$ and $H: Y \longrightarrow 2^{Z}$ such that

$$
H(\operatorname{co}(A)) \subseteq G(A)
$$

for any $A \in\langle Y\rangle$; in this case, $G$ is referred as a generalized KKM mapping with reference to $H$.

If the set-valued mapping $H: Y \longrightarrow 2^{Z}$ satisfies the condition that for any generalized KKM mapping $G: Y \longrightarrow 2^{Z}$ with reference to $H$, the class $\{\overline{G(y)}, y \in Y\}$ has finite intersection property, then $H$ has KKM property, and we write it as follows:

$H \in \operatorname{KKM}(Y, Z)=\left\{H: Y \longrightarrow 2^{Z}, \quad H\right.$ has KKM property $\}$.

Consider the generalized metric space $(W, d)$ and $\phi \neq$ $Y \subset W$. Then, $H: Y \longrightarrow 2^{W}$ is called to have approximate fixed point property if for any $\varepsilon>0$, there exists $y \in Y$ such that

$$
H(y) \cap B(y, \varepsilon) \neq \phi
$$

We now present approximate fixed point property of KKM type mapping on subadmissible subset of a generalized metric space and generalize the main results of $[6,18]$.
Theorem 8. Consider the nonempty subadmissible subset $Y$ of the generalized metric space $(W, d)$. Let $H \in K K M(Y, Y)$ be such that $\overline{H(Y)}$ is totally bounded. Then, $H$ has an approximate fixed point property.

Proof. Consider

$$
Z=\overline{H(Y)} \subset \bar{Y}
$$

where $Z$ is totally bounded. Thus, for any $\varepsilon>0, Y$ has a finite subset $C$ such that

$$
Z \subseteq \cup_{c \in C} B(c, \varepsilon)
$$

where $B(c, \varepsilon)$ is an open ball having radius $\varepsilon$ and center $c$.

Now, we define a map $G: Y \longrightarrow 2^{Y}$ by the following:

$$
G(y)=Z \cap \overline{B^{c}(y, S \varepsilon)}
$$

where $S$ represents the constant associated with inequality and $B^{c}(y, \varepsilon)$ denotes the complement of $B(y, \varepsilon)$ in $W$ for any $\varepsilon>0$ and $y \in Y$. Obviously, $G(y)$ is closed.

Now we prove $\bigcap_{c \in C} G(c)=\phi$. On contrary assume that $\bigcap_{c \in C} G(c) \neq \phi$, then we have the following:

$$
\kappa \in Z \cap \overline{B^{c}(c, S \varepsilon)} \Rightarrow \kappa \in B(c, \varepsilon), \kappa \in \overline{B^{c}(c, S \varepsilon)} .
$$

So, there exists $\left\{\kappa_{n}\right\} \in B^{c}(c, S \varepsilon)$ such that $d\left(\kappa_{n}, \kappa\right)<\varepsilon$ for all $\varepsilon>0$ and $n \geq N$.

So,

$$
\kappa \in B\left(\kappa_{n}, \varepsilon\right) \text { for all } n \geq N \text {. }
$$

Thus,

$$
B\left(\kappa_{n}, \varepsilon\right) \cap B(c, \varepsilon) \neq \phi \text { for all } n \geq N \text {. }
$$

Now, choose $\left\{z_{m_{n}}\right\} \in B\left(\kappa_{n}, \varepsilon\right) \cap B(c, \varepsilon)$ for all $\varepsilon>0$ and $n \geq N$. Then, $\left\{z_{m_{n}}\right\} \in C(d, W, c)$ and

$$
\lim _{n \longrightarrow \infty} d\left(c, \kappa_{n}\right) \leq S \lim _{n, m_{n} \longrightarrow \infty} \sup d\left(z_{m_{n}}, \kappa_{n}\right)<S \varepsilon,
$$

which contradicts to $\left\{\kappa_{n}\right\} \in B^{c}(c, S \varepsilon)$. Thus, we have $\underset{c \in C}{\cap} G$ $(c)=\phi$.

Hence, $G$ is not a generalized KKM mapping with reference to $H$. As $H \in \operatorname{KKM}(Y, Y)$, so there is a finite nonempty subset $D \subseteq Y$ such that

$$
H(\operatorname{co}(D)) \underline{\not \subset} \cup_{\rho \in D} G(\rho)
$$

i.e., we have $y_{\circ} \in H(\operatorname{co}(D))$ such that $y_{\circ} \notin G(\rho)$ for any $\rho \in D$. As $y_{\circ} \notin G(\rho)=Z \cap B^{c}(\rho, S \varepsilon)$, so

$$
y_{\circ} \in\left(\overline{B^{c}(\rho, S \varepsilon)}\right)^{c} \text {, }
$$


for any $\rho \in D$. Now,

$$
y_{\circ} \in\left(\overline{B^{c}(\rho, S \varepsilon)}\right)^{c} \subseteq B(\rho, S \varepsilon),
$$

for any $\rho \in D$. We may write it as $D \subseteq B\left(y_{\circ}, S \varepsilon\right)$.

As

$$
\operatorname{co}(D) \subseteq B\left(y_{\circ}, S \varepsilon\right) .
$$

For $y_{\circ} \in H(\operatorname{co}(D))$, we have $y_{\varepsilon} \in \operatorname{co}(D)$ such that $y_{\circ} \in$ $H\left(y_{\varepsilon}\right)$. And

$$
y_{\varepsilon} \in \operatorname{co}(D) \subseteq B\left(y_{\circ}, S_{\varepsilon}\right) .
$$

Thus, we have the following:

$$
H\left(y_{\varepsilon}\right) \cap B\left(y_{\varepsilon}, S \varepsilon\right) \neq \phi .
$$

As $\varepsilon$ is arbitrary, so, $H$ has an approximate fixed point property.

\section{Applications of KKM Maps}

As the consequence of Theorem 8 , we deduce the following fixed point theorem.

Theorem 9. Consider the nonempty subset $Y$ which is subadmissible in the generalized metric space $(W, d)$ and $H \in$ $\operatorname{KKM}(Y, Y)$ be such that $H$ is compact and closed. Then, $H$ has a fixed point.

Proof. As $H$ is compact, hence, $\overline{H(Y)}$ is compact. So, $\overline{H(Y)}$ is totally bounded. Hence by Theorem $8, H$ has an approximate fixed point property; i.e., for any $\varepsilon>0$, there exists $y_{\varepsilon}$ $\in Y$ such that

$$
H\left(y_{\varepsilon}\right) \cap B\left(y_{\varepsilon}, \varepsilon\right) \neq \phi
$$

In particular for $n \geq 1$ and $\varepsilon=1 / n$, we have $y_{n} \in Y$ such that

$$
H\left(y_{n}\right) \cap B\left(y_{n}, \frac{1}{n}\right) \neq \phi \Rightarrow z_{n} \in H\left(y_{n}\right) \cap B\left(y_{n}, \frac{1}{n}\right) .
$$

Since $\left(z_{n}\right)$ is a sequence in $\overline{H(Y)}$ for $n \geq 1$ and $\overline{H(Y)}$ is compact, so, there exists convergent subsequence $\left(z_{n_{k}}\right)$ in $\overline{H(Y)}$ and suppose it converges to $z$.

Also for $n \geq 1$, we have the following:

$$
d\left(y_{n}, z_{n}\right)<\frac{1}{n} .
$$

As the sequence $\left(z_{n_{k}}\right)$ in $(W, d)$ is convergent to $z$, so, there exists $S>0$ such that

$$
\begin{aligned}
d\left(y_{n}, z\right) & \leq S \lim _{n_{k} \longrightarrow \infty} \sup d\left(y_{n}, z_{n_{k}}\right) \\
& \leq S \lim _{n \longrightarrow \infty} \sup d\left(y_{n}, z_{n}\right) \Rightarrow d\left(y_{n}, z\right) \longrightarrow 0 \text { as } n \longrightarrow \infty
\end{aligned}
$$

So, $\left(y_{n}\right)$ also converges to $z$.

Since $\left\{\left(y_{n}, z_{n}\right)\right\} \in \operatorname{Gr}(H)$ and $H$ is closed, so, $z \in H(z)$, which is our required result.

Example 2. Let $([0, \infty), d)$ be the generalized metric space with a generalized metric:

$$
d(x, y)=(x-y)^{2},
$$

and $Y=[0,1] \subset[0, \infty)$ be subadmissible subset of $([0, \infty), d)$. As $d$ is continuous in first variable from Remark 5.1 in [19], Proposition 3.11 in [20] states that closed ball in $([0, \infty), d)$ is a closed set. Define $H: Y \longrightarrow 2^{Y}$ by the following:

$$
H(y)=[0, y] .
$$

Then, $H$ is a KKM map and $H \in \operatorname{KKM}(Y, Y)$. Also, $H($ $Y)$ is closed and bounded and clearly $\overline{H(Y)}=\overline{[0,1]}$ is totally bounded. So, by Theorem $8, H$ has an approximate fixed point property. Further, $H$ is closed and compact, so by Theorem $9, H$ has a fixed point.

The next result will be helpful to present Schauder's type fixed point theorem for generalized metric spaces.

Lemma 10. Let $Y$ be the nonempty subadmissible subset of the generalized metric space $(W, d)$ and $Z$ be the topological space. Suppose that $g: Z \longrightarrow Y$ is continuous and $H \in$ $K K M(Y, Z)$. Then, $g \circ H \in K K M(Y, Y)$.

Proof. Consider the generalized KKM mapping $G: Y \longrightarrow 2^{Y}$ with reference to $g \circ H$ such that $G(y)$ is closed for every $y$ $\in Y$. Since $G$ is a generalized KKM mapping with reference to $g \circ H$, so, for any nonempty finite subset $A$ of $Y$, we have the following:

$$
g \circ H(c o(A)) \subset \cup_{y \in A} G(y) \Rightarrow H(\operatorname{co}(A)) \subset \cup_{y \in A} g^{-1}(G(y)) .
$$

Thus, $g^{-1}(G)$ is a generalized KKM mapping with reference to $H$.

As $H \in \operatorname{KKM}(Y, Z)$, then $\left\{g^{-1}(G(y)), y \in Y\right\}$ has a finite intersection property.

Also, $g$ is continuous and

$$
\bigcap_{j=1}^{p} g^{-1}\left(G\left(y_{\hat{j}}\right)\right) \neq \phi \Rightarrow g^{-1}\left(\bigcap_{\hat{j}=1}^{p} G\left(y_{\hat{j}}\right)\right) \neq \phi \Rightarrow \bigcap_{\hat{j}=1}^{p} G\left(y_{\hat{j}}\right) \neq \phi .
$$

Thus, the collection $\{G(y), y \in Y\}$ has finite intersection property, which gives $g \circ H \in \operatorname{KKM}(Y, Y)$. 
As the consequence of Theorem 9 and Lemma 10, we obtain Schauder's type fixed point theorem in generalized metric spaces.

Theorem 11. Let $Y$ be the nonempty subadmissible subset of the generalized metric space $(W, d)$. Suppose that $I \in K K M$ $(Y, Y)$. Then any continuous map $S: Y \longrightarrow Y$ such that $S(Y)$ is compact has a fixed point.

Proof. From Lemma 10, we have the following:

$$
S=S \circ I \in \operatorname{KKM}(Y, Y)
$$

As $S$ is continuous and $S(Y)$ is compact, so $S$ is closed and compact. Hence, from Theorem $9, S$ has a fixed point.

Now, we present the generalized Fan's matching theorem in generalized metric spaces by using KKM property.

Theorem 12. Let $Y$ be the nonempty admissible subset of the generalized metric space $(W, d)$ and $Z$ be the topological space. Suppose that $H \in K K M(Y, Z)$ is compact and consider the open valued map $K: Y \longrightarrow 2^{Z}$ satisfying $\overline{H(Y)} \subseteq K(Y)$. Then, there exists $A \in\langle Y\rangle$ such that

$$
H(\operatorname{co}(A)) \cap\left(\cap_{y \in A} K(y)\right) \neq \phi
$$

Proof. Assume that $H \in \operatorname{KKM}(Y, Z)$ and define the multivalued map $G: Y \longrightarrow 2^{Z}$ by $G(y)=\overline{H(Y)} \cap K^{c}(y)$ for $y \in Y$. Then, $G(y)$ is closed for every $y \in Y$. On the contrary, assume that $H(\operatorname{co}(A)) \cap\left(\cap_{y \in A} K(y)\right)=\phi$ for any $A \in\langle Y\rangle$. Since $A \in\langle Y\rangle$ and $Y$ is admissible, so,

$$
\begin{gathered}
c o(A) \subseteq Y \\
\Rightarrow \frac{H(\operatorname{co}(A)) \subseteq \overline{H(Y)}}{\bigcap_{y \in A} K(y)} \\
\Rightarrow H(\operatorname{co}(A)) \subseteq \overline{H(Y)} \cap\left(\bigcap_{y \in A} K(y)\right)^{c} \\
\Rightarrow H(\operatorname{co}(A)) \subseteq \bigcup_{y \in A}\left(\overline{H(Y)} \cap K^{c}(y)\right)=G(A) .
\end{gathered}
$$

Hence, $G$ is a generalized KKM mapping with reference to $H$. As $H \in \operatorname{KKM}(Y, Z)$, thus, the class $\{G(y): y \in Y\}$ has a finite intersection property. So,

$$
\bigcap_{y \in Y} G(y) \neq \phi \Rightarrow y \in \overline{H(Y)} \text { but } y \notin K(Y) \text {, }
$$

which is contrary to the fact $\overline{H(Y)} \subseteq K(Y)$. So, there exists $A \in\langle Y\rangle$ such that

$$
H(\operatorname{co}(A)) \cap\left(\bigcap_{y \in A} K(y)\right) \neq \phi
$$

Now, we present an application of Theorem 11 to find the existence of solutions to the following AB-Caputo fractional BVP:

$$
\left(\begin{array}{cc}
\mathrm{ABC} & \\
0 & D^{\alpha} u
\end{array}\right)(t)=g(t, u(t)), \quad 1<\alpha \leq 2, t \in[0,1]
$$

with boundary conditions

$$
\begin{gathered}
u(0)=0, \\
\lambda u^{\prime}(\eta)=\gamma u^{\prime}(1),
\end{gathered}
$$

where ${ }_{0}^{\mathrm{ABC}} D^{\alpha}$ represents the AB-Caputo fractional derivative and $g:[0,1] \times \mathbb{R} \longrightarrow \mathbb{R}$. Also, $\lambda, \gamma>0,0 \leq t \leq \eta \leq 1$.

Lemma 13 [21]. For $0<\alpha<1$, we obtain the following:

$$
\begin{aligned}
& \left(A_{a} B I^{\alpha} A B C D^{\alpha}\right)(g(t))=g(t)-g(a), \\
& \left(A B I_{b}^{\alpha} A B C D_{b}^{\alpha}\right)(g(t))=g(t)-g(b) .
\end{aligned}
$$

Proposition 14 (see [22]). For $g(t)$ defined on $[a, b]$ and $\alpha$ $\in(\kappa, \kappa+1]$ for some $\kappa \in \mathbb{N}$, we have the following:

$$
\begin{aligned}
& \left(\underset{a}{A B R D^{\alpha}} \underset{a}{A B I^{\alpha}}\right)(g(t))=g(t), \\
& \left(A_{a} B I^{\alpha} A B R D^{\alpha}\right)(g(t))=g(t)-\sum_{\gamma=0}^{\kappa-1} \frac{g^{\gamma}(a)}{\gamma^{!}}(t-a)^{\gamma}, \\
& \left(\underset{a}{A B I^{\alpha} A B C D^{\alpha}}\right)(g(t))=g(t)-\sum_{\gamma=0}^{\kappa} \frac{g^{\gamma}(a)}{\gamma^{!}}(t-a)^{\gamma} .
\end{aligned}
$$

Lemma 15 (see [23]). A subset $H$ in $C(I, \mathbb{R})$ is relatively compact if and only if it is uniformly bounded and equicontinuous on I.

The following lemma will be crucial for the proof of our next result.

Lemma 16. Assume that $K:[0,1] \longrightarrow \mathbb{R}$ is continuous. Then, the solution of linear AB-Caputo fractional BVP

$$
\left(\begin{array}{cc}
A B C & \\
0 & D^{\alpha} u
\end{array}\right)(t)=K(t)
$$

with boundary Equation (45) is given by the following:

$$
u(t)=t \frac{(2-\alpha)}{(\lambda-\gamma) B(\alpha-1)}(\gamma K(1)-\lambda K(\eta))+\int_{0}^{1} G(t, x) K(x) d x
$$


where

$G(t, x)= \begin{cases}\frac{\gamma(\alpha-1)^{2} t}{(\lambda-\gamma) B(\alpha-1) \Gamma(\alpha)}(1-x)^{\alpha-2}-\frac{\lambda(\alpha-1)^{2} t}{(\lambda-\gamma) B(\alpha-1) \Gamma(\alpha)}(\eta-x)^{\alpha-2} & 0 \leq s \leq t, \\ +\frac{(2-a)}{B(\alpha-1)}+\frac{(\alpha-1)}{B(\alpha-1) \Gamma(\alpha)}(t-x)^{\alpha-1} & \\ +\frac{\gamma(\alpha-1)^{2} t}{(\lambda-\gamma) B(\alpha-1) \Gamma(\alpha)}(1-x)^{\alpha-2}-\frac{\lambda(\alpha-1)^{2} t}{(\lambda-\gamma) B(\alpha-1) \Gamma(\alpha)}(\eta-x)^{\alpha-2} & t \leq s \leq \eta, \\ +\frac{\gamma(\alpha-1)^{2} t}{(\lambda-\gamma) B(\alpha-1) \Gamma(\alpha)}(1-x)^{\alpha-2} & \eta \leq s \leq 1 .\end{cases}$

Proof. We have given the following:

$$
\left(\begin{array}{cc}
\mathrm{ABC} & \\
0 & D^{\alpha} u
\end{array}\right)(t)=K(t), \quad 1<\alpha \leq 2, t \in[0,1]
$$

From Proposition 14, we get the following:

$$
\begin{aligned}
u(t)= & c_{1}+c_{2} t+\frac{(2-\alpha)}{B(\alpha-1)} \int_{0}^{t} K(x) d x \\
& +\frac{(\alpha-1)}{B(\alpha-1) \Gamma(\alpha)} \int_{0}^{t}(t-x)^{\alpha-1} K(x) d x
\end{aligned}
$$

Now, using $u(0)=0$ in Equation (52), which implies $c_{1}=0$, replace value of $c_{1}$ in Equation (52):

$$
\begin{aligned}
u(t)= & c_{2} t+\frac{(2-\alpha)}{B(\alpha-1)} \int_{0}^{t} K(x) d x \\
& +\frac{(\alpha-1)}{B(\alpha-1) \Gamma(\alpha)} \int_{0}^{t}(t-x)^{\alpha-1} K(x) d x
\end{aligned}
$$

Take first ordinary derivative on both sides.

$$
\begin{aligned}
u^{\prime}(t)= & c_{2}+\frac{(2-\alpha)}{B(\alpha-1)} K(t) \\
& +\frac{(\alpha-1)}{B(\alpha-1) \Gamma(\alpha)} \int_{0}^{t}(t-x)^{\alpha-2} K(x) d x .
\end{aligned}
$$

Using boundary condition $\lambda u^{\prime}(\eta)=\gamma u^{\prime}$ (35)), in Equation (54), we get the following:

$$
\begin{aligned}
c_{2}= & \frac{(2-\alpha)}{(\lambda-\gamma) B(\alpha-1)}(\gamma K(1)-\lambda K(\eta)) \\
& +\frac{\gamma(\alpha-1)^{2}}{(\lambda-\gamma) B(\alpha-1) \Gamma(\alpha)} \int_{0}^{1}(1-x)^{\alpha-2} K(x) \\
& \cdot d x-\frac{\lambda(\alpha-1)^{2}}{(\lambda-\gamma) B(\alpha-1) \Gamma(\alpha)} \int_{0}^{\eta}(\eta-x)^{\alpha-2} K(x) d x .
\end{aligned}
$$

Putting the value of $c_{2}$ in Equation (53),

$$
\begin{aligned}
u(t)= & t \frac{(2-\alpha)}{(\lambda-\gamma) B(\alpha-1)}(\gamma K(1)-\lambda K(\eta)) \\
& +\frac{\gamma(\alpha-1)^{2} t}{(\lambda-\gamma) B(\alpha-1) \Gamma(\alpha)} \int_{0}^{1}(1-x)^{\alpha-2} K(x) \\
& \cdot d x-\frac{\lambda(\alpha-1)^{2} t}{(\lambda-\gamma) B(\alpha-1) \Gamma(\alpha)} \int_{0}^{\eta}(\eta-x)^{\alpha-2} K(x) \\
& \cdot d x+\frac{(2-\alpha)}{B(\alpha-1)} \int_{0}^{t} K(x) d x+\frac{(\alpha-1)}{B(\alpha-1) \Gamma(\alpha)} \int_{0}^{t}(t-x)^{\alpha-1} K(x) d x .
\end{aligned}
$$

After simplification, we get the required result which is given in Equation (49); i.e.,

$$
u(t)=t+\frac{(2-\alpha)}{(\lambda-\gamma) B(\alpha-1)}(\gamma K(1)-\lambda K(\eta))+\int_{0}^{1} G(t, x) K(x) d x
$$

In the view of Lemma 16, we transform AB-Caputo fractional BVP (Equations (44) and (45)) into a fixed point problem as follows:

$$
u=T u
$$

where the operator $T: C([0,1], \mathbb{R}) \longrightarrow C([0,1], \mathbb{R}) \quad$ is defined as follows:

$$
T(u(t))=\delta_{1}^{*}+\int_{0}^{1} G(t, x) g(x, u(x)) d x
$$

where

$$
\delta_{1}^{*}=t \frac{(2-\alpha)}{(\lambda-\gamma) B(\alpha-1)}(\gamma g(1, u(1))-\lambda g(\eta, u(\eta))),
$$

and $G(t, x)$ is defined in Equation (50).

For now and onwards, take $X=\{u \in C([0,1], \mathbb{R}):|u(t)|$ $<\infty\}$ be the Banach space with norm defined by the following:

$$
\|u\|=\sup _{t \in[0,1]}|u(t)|
$$

We also use following assumptions:

$$
\begin{gathered}
|g(t, u(t))| \leq \mu, \quad \mu>0, \\
\Omega=\frac{2}{(\lambda-\gamma) B(\alpha-1)}[\gamma+\lambda],
\end{gathered}
$$




$$
\begin{aligned}
& \sup _{t \in[0,1]} \int_{0}^{1} G|(t, x)| d x \leq \sup _{t \in[0,1]} \int_{0}^{1}\left\{\left|\frac{\gamma(\alpha-1)^{2} t}{(\lambda-\gamma) B(\alpha-1) \Gamma(\alpha)}(1-x)^{\alpha-2}\right|\right. \\
& +\left|\frac{\lambda(\alpha-1)^{2} t}{(\lambda-\gamma) B(\alpha-1) \Gamma(\alpha)}(\eta-x)^{\alpha-2}\right|+\left|\frac{(2-\alpha)}{B(\alpha-1)}\right| \\
& \left.+\left|\frac{(\alpha-1)}{B(\alpha-1) \Gamma(\alpha)}(1-x)^{\alpha-1}\right|\right\} d x \leq\left|\frac{\gamma(\alpha-1)}{(\lambda-\gamma) B(\alpha-1) \Gamma(\alpha)}\right| \\
& \quad+\left|\frac{\lambda(\alpha-1)}{(\lambda-\gamma) B(\alpha-1) \Gamma(\alpha)}\left(\eta^{\alpha-1}\right)\right|+\left|\frac{(2-\alpha)}{B(\alpha-1)}\right| \\
& \quad+\left|\frac{(\alpha-1)}{B(\alpha-1) \alpha \Gamma(\alpha)}\right|=\mathscr{F} .
\end{aligned}
$$

Remark 17. Since the generalized metric space is not much explored for finding the results like Arzela Ascoli, therefore, in the next application, we will use metric space (as every metric space is a generalized metric space). This application will constitute a base for application of KKM mappings in the existence theory of differential equations.

Theorem 18. Let $X=C([0,1], \mathbb{R}), Y=B[0, r]$, and $I \in K K M$ $(Y, Y)$. Let $g:[0,1] \times \mathbb{R} \longrightarrow \mathbb{R}$ be continuous and satisfying Equations (62)-(64). Then, the AB-Caputo fractional $B V P$ (Equation (44) and Equation (45)) has a solution in $X$.

Proof. $T: X \longrightarrow X$ is defined as follows:

$$
T(u(t))=\delta_{1}^{*}+\int_{0}^{1} G(t, x) g(x, u(x)) d x,
$$

where $\delta_{1}^{*}$ is defined in Equation (60). Suppose a closed ball $Y=B_{r}=\{u \in X:\|u\| \leq r\}$ is a convex subset of $X$. Now consider $u \in B_{r}$ :

$$
\begin{aligned}
& \|T(u(t))\| \leq \sup _{t \in[0,1]}\left\{\left|t \frac{(2-\alpha)}{(\lambda-\gamma) B(\alpha-1)}\right| \gamma g(1, u(1))-\lambda g(\eta, u(\eta)) \mid\right. \\
& \left.\quad+\int_{0}^{1}|G(t, x) g(x, u(x))| d x\right\} \leq \frac{2}{(\lambda-\gamma) B(\alpha-1)} \sup _{t \in[0,1]}\{\gamma \mid(g(1, u(1)) \mid \\
& +\lambda|g(\eta, u(\eta))|\}+\sup _{t \in[0,1]} \int_{0}^{1}|G(t, x) g(x, u(x))| d x \leq \frac{2}{(\lambda-\gamma) B(\alpha-1)} \\
& \cdot[\gamma+\lambda]\|\mu\|+\mathscr{F}\|\mu\|=[\Omega+\mathscr{F}]\|\mu\|,
\end{aligned}
$$

which implies that

$$
\|T(u(t))\| \leq\|\mu\|[\Omega+\mathscr{F}] \leq r .
$$

Hence, $T\left(B_{r}\right) \subseteq B_{r}$.

Continuity of $g$ implies continuity of $T$ and

$$
\|T(u(t))\| \leq r .
$$

Therefore, $T$ is uniformly bounded on $B_{r}$.
Now, we show $T$ is equicontinous. For this, take $0 \leq t_{1}$ $\leq t_{2} \leq \eta \leq 1$.

$$
\begin{aligned}
& \left\|\left(T_{u}\right)\left(t_{2}\right)-(T u)\left(t_{1}\right)\right\|=\sup _{t \in[0,1]} \mid\left\{t_{2} \frac{(2-\alpha)}{(\lambda-\gamma) B(\alpha-1)}\{(\gamma g(1, u(1))\right. \\
& \left.-\lambda g(\eta, u)(\eta))\}+\int_{0}^{1} G\left(t_{2}, x\right) g(x, u(x)) d x\right\} \\
& -\left\{\begin{array}{c}
t_{1} \frac{(2-\alpha)}{(\lambda-\gamma) B(\alpha-1)}\{(\gamma g(1, u(1))-\lambda g(\eta, u)(\eta))\} \\
+\int_{0}^{1} G\left(t_{1}, x\right) g(x, u(x)) d x
\end{array}\right\} \\
& =\sup _{t \in[0,1]} \mid\left\{\left(t_{2}-t_{1}\right) \frac{(2-\alpha)}{(\lambda-\gamma) B(\alpha-1)}\{(\gamma g(1, u(1))-\lambda g(\eta, u)(\eta))\} d x\right\} \\
& +\left\{\int_{0}^{1}\left\{G\left(t_{2}, x\right)-G\left(t_{1}, x\right)\right\} g(x, u(x)) d x\right\} \mid \\
& \leq \frac{2}{(\lambda-\gamma) B(\alpha-1)} \sup _{t \in[0,1]}\left[\mid\left\{\left(t_{2}-t_{1}\right)\{(\gamma g(1, u(1))-\lambda g(\eta, u(\eta))\}\} \mid\right.\right. \\
& \left.\quad+\int_{0}^{1}\left|\left\{G\left(t_{2}, x\right)-G\left(t_{1}, x\right)\right\} \| g(x, u(x))\right| d x\right] \longrightarrow 0 \text { as } t_{1} \longrightarrow t_{2} .
\end{aligned}
$$

Therefore, $T$ is equicontinuous. Further by virtue of Lemma $15, \overline{T(B)}$ is compact. Therefore, by Theorem 11 , $T$ has a fixed point in $Y$, which means given $A B$-Caputo fractional BVP (Equation (44) and Equation (45)) has a solution.

\section{Data Availability}

No data is used in this paper.

\section{Conflicts of Interest}

The authors declare that they have no conflicts of interest.

\section{References}

[1] R. P. Agarwal, N. Hussain, and M. A. Taoudi, "Fixed point theorems in ordered Banach spaces and applications to nonlinear integral equations," Abstract and Applied Analysis, vol. 2012, 15 pages, 2012.

[2] M. S. Ashraf, R. Ali, and N. Hussain, "New fuzzy fixed point results in generalized fuzzy metric spaces with application to integral equations," IEEE Access, vol. 8, pp. 91653-91660, 2020.

[3] M. Younis, D. Singh, I. Altun, and V. Chauhan, "Graphical structure of extended b-metric spaces: an application to the transverse oscillations of a homogeneous bar," International Journal of Nonlinear Sciences and Numerical Simulation, 2021.

[4] S. Czerwik, "Contraction mappings in b-metric spaces," Acta Mathematica et Informatica Universitatis Ostraviensis, vol. 1, no. 1, pp. 5-11, 1993.

[5] S. Czerwik, Nonlinear Set-Valued Contraction Mappings in bMetric Spaces, 1998.

[6] M. A. Khamsi and N. Hussain, "KKM mappings in metric type spaces," Nonlinear Analysis: Theory, Methods \& Applications, vol. 73, no. 9, pp. 3123-3129, 2010. 
[7] P. Hitzler and A. K. Seda, "Dislocated topologies," Journal of Electrical Engineering, vol. 51, no. 12, pp. 3-7, 2000.

[8] M. Jleli and B. Samet, "A generalized metric space and related fixed point theorems," Fixed Point Theory and Applications, vol. 2015, no. 1, 14 pages, 2015.

[9] B. Knaster, C. Kuratowski, and S. Mazurkiewicz, "Ein Beweis des Fixpunktsatzes für n-dimensionale Simplexe," Fundamenta Mathematicae, vol. 14, no. 1, pp. 132-137, 1929.

[10] K. Fan, "Fixed-point and minimax theorems in locally convex topological linear spaces," Proceedings of the National Academy of Sciences of the United States of America, vol. 38, no. 2, pp. 121-126, 1952.

[11] A. Granas, "KKM-maps and their applications to nonlinear problems," The Scottish Book, pp. 45-61, 1981.

[12] S. Singh, B. Watson, and P. Srivstana, Fixed Point Theory and Best Approximation, The KKM-map Principle Kluwer Academic Publishers, 1997.

[13] G. X. Z. Yuan, KKM Theory and Applications in Nonlinear Analysis, vol. 218, CRC Press, 1999.

[14] N. Hussain and M. H. Shah, "KKM mappings in cone b-metric spaces," Computers \& Mathematics with Applications, vol. 62, no. 4, pp. 1677-1684, 2011.

[15] K. Fan, "A generalization of Tychonoff's fixed point theorem," Mathematische Annalen, vol. 142, no. 3, pp. 305-310, 1961.

[16] M. A. Khamsi, "KKM and Ky Fan theorems in hyperconvex metric spaces," Journal of Mathematical Analysis and Applications, vol. 204, no. 1, pp. 298-306, 1996.

[17] T. H. Chang and C. L. Yen, "KKM property and fixed point theorems," Journal of Mathematical Analysis and Applications, vol. 203, no. 1, pp. 224-235, 1996.

[18] A. Amini, M. Fakhar, and J. Zafarani, "KKM mappings in metric spaces," Nonlinear Analysis: Theory, Methods \& Applications, vol. 60, no. 6, pp. 1045-1052, 2005.

[19] M. Akkouchi, "A common fixed point theorem for expansive mappings under strict implicit conditions on b-metric spaces," Acta Universitatis Palackianae Olomucensis. Facultas Rerum Naturalium. Mathematica, vol. 50, no. 1, pp. 5-15, 2011.

[20] T. Van An, L. Q. Tuyen, and N. Van Dung, "Stone-type theorem on b-metric spaces and applications," Topology and its Applications, vol. 185-186, pp. 50-64, 2015.

[21] T. Abdeljawad and D. Baleanu, "Discrete fractional differences with nonsingular discrete Mittag-Leffler kernels," Advances in Difference Equations, vol. 2016, no. 1, 2016.

[22] T. Abdeljawad, "A Lyapunov type inequality for fractional operators with nonsingular Mittag-Leffler kernel," Journal of Inequalities and Applications, vol. 2017, no. 1, 11 pages, 2017.

[23] Y. Zhou, J. Wang, and L. Zhang, Basic Theory of Fractional Differential Equations, World Scientific, 2016. 